\title{
Análise dos Resíduos Madeireiros Gerados Pelas Serrarias do Município de Eunápolis-BA
}

\author{
Pedro Henrique Alcântara de Cerqueira ${ }^{1}$, Giovanni Correia Vieira ${ }^{1}$, \\ Iara Magalhães Barberena ${ }^{1}$, Lara Clímaco Melo ${ }^{1}$, Luís Carlos de Freitas ${ }^{1}$ \\ ${ }^{1}$ Departamento de Fitotecnia e Zootecnia - DFZ, Universidade Estadual do Sudoeste da Bahia - UESB, \\ Vitória da Conquista/BA, Brasil
}

\begin{abstract}
RESUMO
Com a crescente demanda de madeira serrada nos últimos anos, o estudo da cadeia produtiva do segmento é de fundamental importância para a melhor gestão desse setor. Dessa forma, o objetivo deste trabalho foi caracterizar os resíduos gerados pelo processamento ou beneficiamento dos produtos madeireiros comercializados no município de Eunápolis-BA. Os dados foram obtidos por meio de entrevistas, com aplicação de formulários aos responsáveis em todas as serrarias do município. Os aspectos observados foram quanto à utilização de seus resíduos, bem como a sua destinação final. Observou-se resíduos nas serrarias do município de Eunápolis em torno de $165 \mathrm{~m}^{3}$ mensais, sendo $55 \%$ destes destinados para fins energéticos. Neste estudo, foi notada a necessidade de abordagens mais detalhadas sobre o aproveitamento e a maior agregação de valor para os resíduos gerados pelas serrarias.
\end{abstract}

Palavras-chave: produtos madeireiros, matéria-prima florestal, resíduo madeireiro.

\section{Analysis of Sawmill Wood Waste Generated by Mills in Eunapolis, State of Bahia, Brazil}

\begin{abstract}
With the growing demand for lumber in recent years, the study of the production chain of this segment is of fundamental importance for the better management of the sector. Therefore, the objective of this study was to characterize the wood waste generated from the processing of timber products sold in Eunapolis, State of Bahia. Data were obtained by means of interviews with questionnaires applied to those responsible for all logging activitiesin the municipality.The use of woodwasteand its final destination were the main features observed. The waste accumulation of themills in Eunapolis is around 165 cubic meters per month, 55\% of whichintended for energy purposes. This study uncovered the need for more detailed information on the utilization and higher added value of the waste generated by sawmills.
\end{abstract}

Keywords: wood products, forest raw material, timber waste.

\section{INTRODUÇÃO}

Com a crescente demanda de madeira serrada nos últimos anos, o estudo da cadeia produtiva do segmento passou a receber uma atenção especial.
Os produtos oriundos da indústria de base florestal são os mais variados, podendo ser divididos em oito cadeias produtivas principais: chapas e compensados; 
óleos e resinas; fármacos; cosméticos; alimentos; carvão, lenha e energia; papel e celulose; madeira e móveis (SBS, 2007).

Independentemente do tipo de indústria madeireira, a geração de resíduos é consequência direta do processamento primário ou secundário da madeira sólida. Segundo Fontes (1994), de acordo com suas características morfológicas, os resíduos dessas indústrias são classificados como: cavacos - partículas com dimensões máximas de $50 \times 20 \mathrm{~mm}$, em geral provenientes do uso de picadores; maravalhas - resíduo com menos de 2,5 mm; serragem - partículas de madeira com dimensões entre 0,5 e 2,5 $\mathrm{mm}$, provenientes do uso de serras; pó - resíduos menores que 0,5 mm; lenha - resíduos de maiores dimensões, compostos por costaneiras, aparas e resíduo de topo de tora.

A maioria dos resíduos da cadeia produtiva madeireira é gerada no processamento primário, embora a fração percentual que representa os resíduos varie em função de determinados fatores, como o tipo de processo, as máquinas utilizadas e as dimensões e características físicas e anatômicas das toras. Em geral, as perdas no desdobro e nos cortes de resserra de madeira de reflorestamento situam-se entre $20 \%$ e $40 \%$ do volume das toras processadas (Finotti et al., 2006).

A questão do resíduo florestal na indústria é muito discutida, pois o volume de perdas e o não aproveitamento são considerados muito grande, mesmo em marcenarias e pequenas serrarias (Mady, 2000).

Os resíduos gerados pela indústria madeireira é um material que constitui uma ameaça para o meio ambiente quando disposto de forma inadequada. Esse material, porém, pode ser utilizado de maneira alternativa para incrementar as receitas de alguns estabelecimentos madeireiros (Franceschini, 2004).

$\mathrm{O}$ aproveitamento de resíduos de madeira tem contribuído para a racionalização dos recursos florestais, proporcionando uma nova alternativa socioeconômica às empresas, além de contribuir para uma adequação ambiental do gerenciamento de resíduos sólidos industriais.

Dessa forma, a presente pesquisa teve como objetivo a caracterização dos resíduos sólidos das serrarias do município de Eunápolis-BA, por meio da identificação e da quantificação, bem como oferecer sugestões de medidas para melhor aproveitamento desse material.

\section{MATERIAL E MÉTODOS}

Os dados foram coletados em serrarias no município de Eunápolis-Ba, localizado no extremo sul do Estado, situado a $16^{\circ} 26^{\prime}$ de latitude sul e $39^{\circ} 05^{\prime}$ de longitude oeste, no período de 16 a 20 de janeiro de 2011 (Figura 1).

Foram avaliadas todas as serrarias com alvará de funcionamento expedido pela prefeitura do município, totalizando cinco empresas. Para o levantamento das informações referentes aos resíduos madeireiros, elaborou-se um formulário de pesquisa, utilizado nas visitas e aplicado em forma de entrevista individual com os representantes de cada empresa, no próprio estabelecimento. O formulário para avaliação foi estruturado com as seguintes informações: características cadastrais das serrarias; tipos de matéria-prima; origem da madeira; equipamentos utilizados e o respectivo ano de fabricação; consumo médio de madeira por mês; principais produtos fabricados; quantidade média de resíduos gerados mensalmente; máquinas geradoras de resíduos, e a destinação final dos resíduos.

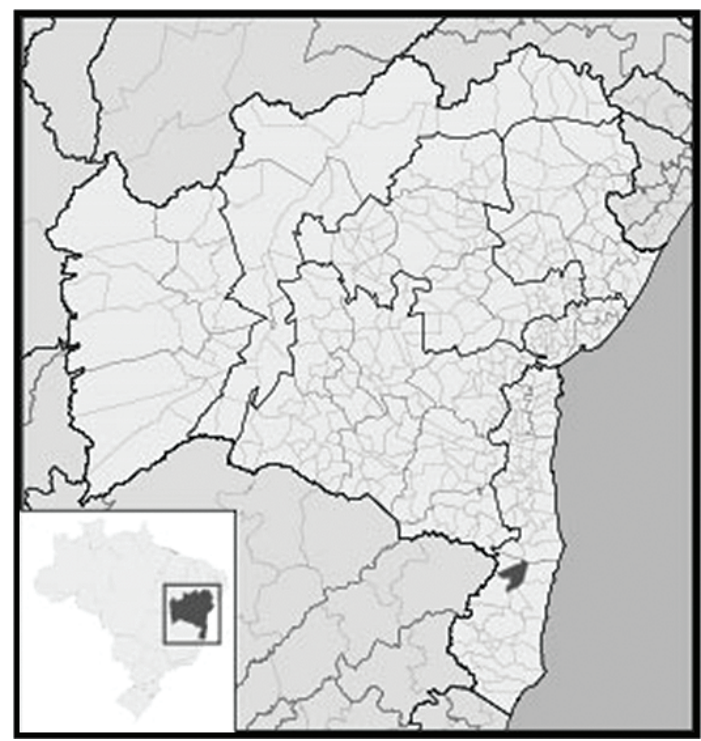

Figura 1. Localização de Eunápolis-BA.

Figure 1. Location of the Eunápolis-BA municipality. 


\section{RESULTADOS E DISCUSSÃO}

O tempo de atuação das empresas no mercado perfaz, em média, 14 anos, com um número de funcionários variando de 10 a 25 . A madeira utilizada pelos estabelecimentos é preponderantemente eucalipto (100\%), sendo este originado em sua totalidade do município de Eunápolis e das regiões circunvizinhas. Em relação à produção de matériaprima, apenas (17\%) possuem plantio próprio para suprimento de madeira; os outros (83\%) adquirem a madeira por meio de terceiros.

Os produtos fabricados pelas serrarias consistem em embalagens para armazenamento de frutas (57\%), pranchas e vigas (21\%), pequenos artefatos (12\%) e móveis (10\%). Entre os principais equipamentos presentes nas serrarias, os que mais geram resíduos, segundo os entrevistados, são a serra de fita (68\%), o traçador (17\%), a serra multilâmina (9\%) e a lixadeira (6\%). Com relação ao tempo de uso dos equipamentos, a grande maioria é considerada obsoleta, sendo que $56 \%$ possuem idade entre 21 e 30 anos, $27 \%$ com idade entre 11 e 20 anos, e $17 \%$ com idade de zero a dez anos. De acordo com os entrevistados, apenas 16\% realizam manutenção periódica dos equipamentos.

Constatou-se que os principais resíduos gerados pelas serrarias são serragem $(36,17 \%)$, lenha (25,53\%), maravalhas $(23,40 \%)$ e cavacos $(12,77 \%)$. Em relação à destinação dos resíduos gerados pelas serrarias avaliadas, constatou-se que a maioria das empresas (55\%) realiza a venda dos resíduos para a geração de energia em cerâmicas próximas ao município, $17 \%$ realizam a venda dos resíduos para utilização em baias de animal, 16\% doam a interessados, $8 \%$ descartam nos lixões e $2 \%$ utilizam para confecção de pequenos artefatos de madeira (Figura 2).

O município de Eunápolis e outras cidades da região sul e do extremo sul da Bahia é um dos principais polos florestais brasileiros, com extensos plantios de eucalipto para suprir indústrias do segmento de celulose e papel instaladas na região. Tal fator permite compreender a utilização apenas da madeira de eucalipto nas serrarias avaliadas. Além da eucaliptocultura, a região também se destaca pela fruticultura, por meio da cultura do mamão. Na presente pesquisa, o principal produto fabricado nas serrarias são justamente embalagens para armazenamento do mamão, fato que se justifica pela demanda existente para a exportação e a comercialização interna da fruta.

As empresas pesquisadas apresentaram um beneficiamento mensal médio de $105 \mathrm{~m}^{3}$ de madeira, gerando cerca $40 \mathrm{~m}^{3}$ de resíduos mensais cada uma. Anualmente, esse valor é de $1.720 \mathrm{~m}^{3}$ de madeira processada e 396,6 $\mathrm{m}^{3}$ de resíduos gerados por cada serraria (Tabela 1). Em relação ao rendimento operacional, caracterizado por Rocha (1999) como a relação entre o volume de toras serradas num período ou turno e o volume de madeira serrada obtido destas toras, as empresas avaliadas apresentaram um rendimento operacional médio de $68,6 \%$.

Tal rendimento condiz com estudos apresentados por Finotti et al. (2006), os quais encontraram rendimento operacional médio das serrarias brasileiras na faixa de $60 \%$ a $80 \%$, configurando um cenário de baixo nível tecnológico e mau aproveitamento da madeira em função da grande quantidade de resíduos gerados.

O conhecimento dos resíduos gerados por uma empresa tem importância tanto econômica quanto ambiental, pois se espera maior retorno econômico durante todo o processo produtivo e menor impacto ambiental. Tais aspectos podem ser amplamente resolvidos por meio do aproveitamento máximo dos resíduos gerados durante toda a cadeia produtiva (Mendoza et al., 2010).

Segundo Cassilha et al. (2004), os resíduos da madeira podem ser utilizados na forração de granjas, currais e baias, assim como na agricultura, para aumentar a retenção de umidade do solo, uma vez que a maioria desses resíduos não possui substancias tóxicas ao meio ambiente. Diversamente, Gomes \& Sampaio (2004) afirmam que o processo de compostagem, geração de energia e fabricação de pequenos artefatos de madeira representa as principais alternativas para o gerenciamento dos resíduos de madeira.

A grande parte da destinação final dos resíduos gerados pelas serrarias do município de Eunápolis está inserida nas principais recomendações descritas na literatura, apresentando apenas uma pequena taxa (8\%) de descarte em lixões do município. Resultado 
semelhante foi encontrado num trabalho realizado por Vieira et al. (2009), no município de Barra do Choça-BA.

Nesse contexto, uma melhor gestão dos resíduos sólidos do setor madeireiro se faz necessária, pois, no
Brasil, uma grande quantidade de resíduos florestais é gerada anualmente pelas diversas indústrias de base florestal. Um exemplo pode ser dado: a geração de resíduos na cadeia produtiva de serrados de Pinus, que é da ordem de 75\%, ou seja, apenas

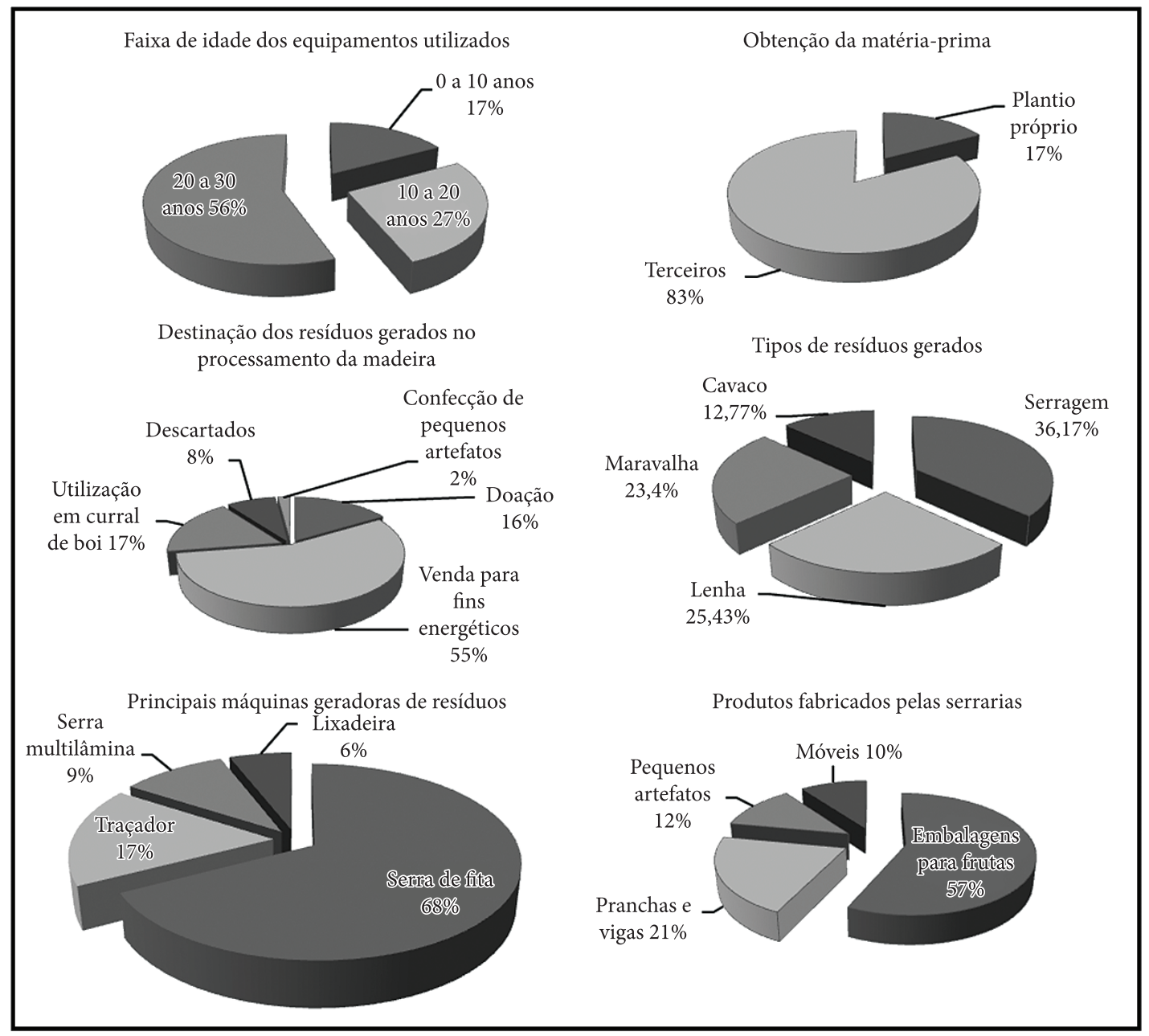

Figura 2. Caracterização das atividades relacionadas aos resíduos sólidos das serrarias.

Figure 2. Characterization of the activities related to solid waste from sawmills.

Tabela 1. Representação do consumo e da geração de resíduos de madeira em cinco serrarias avaliadas no município de Eunápolis-BA.

Table 1. Representation of consumption and waste generation of timber valued at five sawmills in Eunápolis-BA.

\begin{tabular}{cccccc} 
Empresa & $\begin{array}{c}\text { Quantidade } \\
\text { serrada }\left(\mathbf{m}^{\mathbf{3}} \mathbf{m} \mathbf{m} \mathbf{s}\right)\end{array}$ & $\begin{array}{c}\text { Resíduos gerados } \\
\left(\mathbf{m}^{3} / \mathbf{m e ̂ s}\right)\end{array}$ & $\begin{array}{c}\text { Quantidade } \\
\text { serrada }\left(\mathbf{m}^{\mathbf{3}} / \mathbf{a n o}\right)\end{array}$ & $\begin{array}{c}\text { Resíduos gerados } \\
\left(\mathbf{m}^{3} / \mathbf{a n o}\right)\end{array}$ & $\begin{array}{c}\text { Aproveitamento } \\
\text { médio }(\%)\end{array}$ \\
\hline A & 110,0 & 38,5 & 1320,0 & 462,0 & 65 \\
B & 90,0 & 24,3 & 1080,0 & 291,6 & 73 \\
C & 85,0 & 25,5 & 1020,0 & 306,0 & 70 \\
D & 140,0 & 42,0 & 1680,0 & 504,0 & 70 \\
E & 100,0 & 35,0 & 3500,0 & 420,0 & 65 \\
\hline Total & 525,0 & 165,3 & 8600,0 & 1983,0 & $68,6 \%$ \\
\hline Média & $105,0 \mathrm{~m}^{3}$ & $33,0 \mathrm{~m}^{3}$ & $1720,0 \mathrm{~m}^{3}$ & $396,6 \mathrm{~m}^{3}$ & \\
\hline
\end{tabular}


$25 \%$ do volume total de uma árvore é colocado no mercado na forma de tábuas, caibros e ripas (CAF, 2005). A partir desse contexto, deve-se buscar, como uma das alternativas, a estruturação de pequenas cooperativas na cidade, constituindo-se esta uma forma de potencializar os aspectos socioeconômicos da região, com a geração de emprego e renda, e buscando, sobretudo, a otimização dos resíduos florestais.

\section{CONCLUSÕES}

De acordo com os resultados, concluiu-se que há novos usos potenciais para o aproveitamento dos resíduos produzidos pelas serrarias, sobretudo aqueles referentes à produção de novos produtos. Dentre estes, há o adubo orgânico, por meio da compostagem, e a fabricação de pequenos objetos de madeira, contribuindo para a diversificação dos produtos gerados e o fortalecimento da atividade madeireira no município de Eunápolis. Dessa forma, a criação de cooperativas poderá viabilizar técnica e economicamente a utilização destes recursos.

\section{STATUS DA SUBMISSÃO}

Recebido: $19 / 04 / 2012$

Aceito: $20 / 08 / 2012$

Publicado: 31/12/2012

\section{AUTOR(ES) PARA CORRESPONDÊNCIA}

\section{Pedro Henrique Alcântara de Cerqueira}

Faculdade de Engenharia Florestal, Universidade Estadual do Sudoeste da Bahia - UESB, Estrada do Bem-querer, $\mathrm{Km} \mathrm{4}$, Bem-querer, CEP 45083-900, Vitória da Conquista, BA, Brasil e-mail: pedrohenrique.alc@gmail.com

\section{Giovanni Correia Vieira}

Faculdade de Engenharia Florestal, Universidade Estadual do Sudoeste da Bahia - UESB, Estrada do Bem-querer, $\mathrm{Km} 4$, Bem-querer, CEP 45083-900, Vitória da Conquista, BA, Brasil e-mail: cvgiovanni@gmail.com

\section{REFERENNCIAS}

Cassilha AC, Podlasek CL, Casagrande Junior EF, Silva MC, Mengatto SNF. Indústria moveleira e resíduos sólidos: considerações para o equilíbrio ambiental. Revista Educação \& Tecnologia 2004; 8: 209-228.

Consultoria Agro Florestal - CAF. Energia a partir de resíduos florestais. [cited 2005]. Available from: http:// www.cafltda.com.br/01/2005_01.htm

FInotti AR, Schneider VE, Wander PR, Hillig E, Silva MD’A. Uso energético de resíduos de madeira na cadeia produtiva de madeira/móveis e possibilidades de geração de créditos de carbono. In: Pólo Moveleiro da Serra Gaúcha. Sistemas de gerenciamento ambiental na indústria moveleira. EdUCS; 2006.

Fontes PJP. Auto-Suficiência Energética em Serraria de Pinus e Aproveitamento dos Resíduos [dissertação]. Curitiba: Universidade Federal do Paraná; 1994.

Franceschin GL. Biomassa de madeira pode gerar 28 $M W$ de energia. FSP Saneamento \& Meio Ambiente [cited 2004 abr. 20]. Available from: http://pib. socioambiental.org $/ \mathrm{c} /$ noticias? $\mathrm{id}=32081$.

Gomes JI,Sampaio SS. Aproveitamento de resíduos de Madeira em Três Empresas Madeireiras do Estado do Pará. Belém: Embrapa; 2004. Comunicado técnico, n. 102.

Mady FTM. Conhecendo a madeira: informações sobre 90 espécies comerciais. Manaus: SEBRAE; 2000. Programa de Desenvolvimento Tecnológico.

Mendoza ZMSH, Evangelista WV, Araújo SO, Souza CC, Ribeiro FDL, Silva CJ. Análise dos resíduos madeireiros gerados nas marcenarias do município de Viçosa - Minas Gerais. Revista Árvore 2010; 34(4): 755-760. http://dx.doi.org/10.1590/ S0100-67622010000400020

Rocha MP. Desdobro primário da madeira. Curitiba: Fupef; 1999. Série Didática, n. 02/99.

Sociedade Brasileira de Silvicultura - SBS. Fatos e números do Brasil florestal. São Paulo, 2007. 110 p.

Vieira GC, Ribas MCA, Pereira Neto A, Silva GC. Matéria-Prima e Resíduos na Industrialização de Produtos Madeireiros no Município de Barra do Choça - BA. In: Anais do I Seminário Florestal do Sudoeste da Bahia; 2009; Vitória da Conquista. Vitória da Conquista: UESB; 2009. p. 121-124. 